\title{
BMJ Open Impact of low-dose CT screening for lung cancer on ethnic health inequities in New Zealand: a cost- effectiveness analysis
}

\author{
Melissa McLeod (D , ${ }^{1}$ Peter Sandiford, ${ }^{2,3}$ Giorgi Kvizhinadze, ${ }^{4}$ Karen Bartholomew, ${ }^{2}$ \\ Sue Crengle (1D ${ }^{5}$
}

To cite: McLeod M, Sandiford P, Kvizhinadze G, et al. Impact of low-dose CT screening for lung cancer on ethnic health inequities in New Zealand: a cost-effectiveness analysis. BMJ Open 2020;10:e37145. doi:10.1136/ bmjopen-2020-037145

- Prepublication history for this paper is available online. To view these files, please visit the journal online (http://dx.doi org/10.1136/bmjopen-2020037145).

Received 20 January 2020 Revised 30 July 2020 Accepted 14 August 2020
D) Check for updates

(C) Author(s) (or their employer(s)) 2020. Re-use permitted under CC BY-NC. No commercial re-use. See rights and permissions. Published by BMJ.

${ }^{1}$ Department of Public Health, University of Otago, Wellington, New Zealand

${ }^{2}$ Waitemata District Health Board, Takapuna, New Zealand ${ }^{3}$ Auckland District Health Board, Auckland, New Zealand

${ }^{4}$ Wellington, New Zealand

${ }^{5}$ Department of Preventive and Social Medicine, University of Otago, Dunedin, New Zealand

Correspondence to

Dr Sue Crengle;

sue.crengle@otago.ac.nz

\section{ABSTRACT}

Objective There are large inequities in the lung cancer burden for the Indigenous Māori population of New Zealand. We model the potential lifetime health gains, equity impacts and cost-effectiveness of a national lowdose CT (LDCT) screening programme for lung cancer in smokers aged 55-74 years with a 30 pack-year history, and for formers smokers who have quit within the last 15 years.

Design A Markov macrosimulation model estimated: health benefits (health-adjusted life-years (HALYs)), costs and cost-effectiveness of biennial LDCT screening. Input parameters came from literature and NZ-linked health datasets.

Setting New Zealand.

Participants Population aged 55-74 years in 2011. Interventions Biennial LDCT screening for lung cancer compared with usual care.

Outcome measures Incremental cost-effectiveness ratios were calculated using the average difference in costs and HALYs between the screened and the unscreened populations. Equity analyses included substituting nonMāori values for Māori values of background morbidity, mortality and stage-specific survival. Changes in inequities in lung cancer survival and 'health-adjusted life expectancy' (HALE) were measured.

Results LDCT screening in NZ is likely to be cost-effective for the total population: NZ\$34400 per HALY gained (95\% uncertainty interval NZ\$27500 to NZ\$42900) and for Māori separately (using a threshold of gross domestic product per capita NZ\$45000). Health gains per capita for Māori females were twice that for non-Māori females and $25 \%$ greater for Māori males compared with non-Māori males. LDCT screening will narrow absolute inequities in HALE and lung cancer mortality for Māori, but will slightly increase relative inequities in mortality from lung cancer (compared with non-Māori) due to differential stagespecific survival.

Conclusion A national biennial LDCT lung cancer screening programme in New Zealand is likely to be costeffective, will improve total population health and reduce health inequities for Māori. Attention must be paid to addressing ethnic inequities in stage-specific lung cancer survival.
Strengths and limitations of this study

- This new analysis of lung cancer screening updates a previously published model to incorporate parameters and assumptions drawn from the NELSON low-dose CT (LDCT) screening trial.

- There is specific consideration of the impact of lung cancer screening on equity in health outcomes.

- The model allows for the future unrelated health costs of those whose lifespan is extended by the screening.

- Until a pilot of LDCT screening is conducted in New Zealand, estimates of some parameters will be subject to some uncertainty.

\section{INTRODUCTION}

Lung cancer causes $18.3 \%$ of cancer deaths in New Zealand (NZ) - more than any other tumour type-with stark inequities in lung cancer incidence and mortality between Māori (the Indigenous population) and nonMāori. ${ }^{1}$ In 2016 the Māori age-standardised new registration rate for lung cancer was more than triple the rate in non-Māori (77.8 vs 22.4 per 100000 , respectively). ${ }^{2}$ The mortality rate from lung cancer is also more than three times higher in Māori than nonMāori. ${ }^{1}$ Lung cancer survival in NZ is poorer than that of several other countries. ${ }^{34}$

International evidence from a number of large randomised controlled trials (RCTs) shows that low-dose CT (LDCT) screening for lung cancer reduces lung cancer mortality ${ }^{5-9}$ and can also be cost-effective if stringent riskbased eligibility criteria are employed. ${ }^{10-14}$ As the evidence base for LDCT lung cancer screening evolves with improvements to the screening protocols, the likely impacts and cost-effectiveness of such a programme in the NZ context have changed. The most recent evidence of efficacy comes from the Nederlands-Leuvens Longkanker Screenings 
Onderzoek (NELSON RCT) which found that LDCT screening for lung cancer decreased lung cancer mortality by $24 \%$ in high-risk men and $33 \%$ in high-risk women over a 10 -year period. ${ }^{8}$ In comparison, the earlier National Lung Screening Trial (NLST) study found an overall $20 \%$ reduction in lung cancer mortality, ${ }^{5}$ although a more recent analysis with longer follow-up found only an $11 \%$ reduction. ${ }^{9}$ The higher effectiveness observed in the NELSON trial has been attributed to improvements in nodule management through monitoring of nodule volumes and volume doubling times. This improved the specificity of LDCT screening and reduced the costs and harms from diagnostic tests, with a small increase in the number of CT scans required. ${ }^{15}$ Given the higher proportion of distant disease at diagnosis in NZ compared with these large RCT cohorts, there is potential for lung cancer LDCT screening to have an even greater impact on lung cancer mortality than that measured in these large RCTs. ${ }^{16}$

Existing analyses of LDCT lung screening costeffectiveness in the NZ setting ${ }^{17}{ }^{18}$ do not take into account developments in the evidence base (in particular new evidence from the NELSON trial and new analyses of stage at diagnosis and stage-specific survival) and do not explicitly model the impacts of an LDCT lung screening programme on health equity (for Māori). Given the NZ Government's obligations under the Treaty of Waitangi ${ }^{19}$ and the Ministry of Health's stated commitment to health equity, such information is critical in assessing the appropriateness of LDCT lung cancer screening in the NZ setting.

A screening programme that can reduce the burden of lung cancer for those at risk due to high smoking exposure offers great potential to address the huge burden of lung cancer for Māori in NZ. Although progress has been made in tobacco control in NZ, driven by a suite of tobacco control strategies over the last 30 years, ${ }^{20} 35 \%$ of Māori adults continue to smoke compared with $16 \%$ of non-Māori adults. The health burden from tobacco use falls more heavily on Māori who suffer higher incidence and mortality from the majority of tobacco-related diseases $^{21}{ }^{22}$ including lung cancer.

With health equity for Māori as our primary concern, the objectives of this study were (1) to estimate health gains and cost-effectiveness of an LDCT screening programme for lung cancer in Māori smokers aged 55-74years with a 30 pack-year smoking history (Māori and non-Māori) and (2) to measure the impact of LDCT screening on absolute inequalities in lung cancer mortality and healthadjusted life expectancy (HALE) for Māori compared with non-Māori.

\section{MATERIALS AND METHODS}

This study used an existing LDCT lung cancer screening Markov model developed by the University of Otago Burden of Disease Epidemiology, Equity and CostEffectiveness Programme $\left(\mathrm{BODE}^{3}\right)$ that was funded by

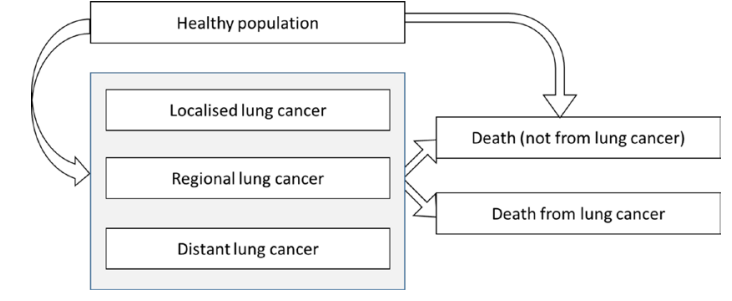

Figure 1 Model states.

the Health Research Council and Ministry of Business, Innovation and Employment. ${ }^{23}$ The lung cancer Markov model feeds cancer-specific mortality to a life table to calculate health-adjusted life-years (HALYs) and costs over a lifetime time horizon. We have updated the original $\mathrm{BODE}^{3}$ model to account for developments in lung cancer screening pathways, to include the most recent RCT evidence and lung cancer epidemiology, while reviewing and revising key model assumptions (described in online supplemental data A, methods).

The updated model (now referred to as 'the model') was used to estimate the lifetime health gains in HALYs, costs and cost-effectiveness of a national LDCT lung screening programme based on biennial CTs of current smokers aged 55-74years with a 30 pack-year smoking history, and of former smokers who have quit within the last 15 years. The same population was modelled twice: without LDCT screening and with LDCT screening for lung cancer. The differences between the two determined benefits and costs.

Included in the model were all individuals aged 55 years and over in 2011 (with the smoking history described above), with each 5-year age group in 2011 modelled separately until death or age 110 years (figure 1 ). From the first round our cohort was diverse, covering the range of eligible age groups with a mix of eligible smoking histories. We assumed this population to be screen-naive. A cycle length of 1 year was used in the modelling. The model was implemented in Microsoft Excel, using the Ersatz add-in for uncertainty analysis (Microsoft, www. Epigear.com).

As much as possible we captured sociodemographic heterogeneity by sex, age and ethnic group (Māori and non-Māori) for lung cancer incidence, stage at diagnosis and mortality. Background mortality, morbidity, and unrelated and disease-specific health system costs were included by sex and age only. Unrelated health system costs were included, that is, average expected costs to the health system by sex and age, generating cost off-sets, for years of life saved. A health system perspective was used, with a 3\% discount rate on both costs and benefits. We assumed a cost-effectiveness threshold value based on annual gross domestic product (GDP) per capita of NZ\$45000 per HALY. ${ }^{24}$

\section{Identification of the modelled population}

The modelled population included those with a 30 packyear history of smoking who either currently smoke or 
have quit in the last 15 years. The eligible population was estimated from NZ census data on current, former and never smokers and smoking cessation rates applied to population estimates. ${ }^{25}$ There was no inflow into the model for those reaching the 30 pack-year criterion after the original cohort was identified. A smoking cessation rate (based on 2013 estimates) ${ }^{25}$ was applied to the modelled population to estimate the proportion of the original cohort becoming ineligible as a result of being more than 15 years post-quitting (model outflow). After quitting, ex-smokers' risk of lung cancer was projected to reduce steadily over time, with no increased risk (over never smokers) at 15 years. In the model, lung cancer cases remaining in the prevalent pool after 6 years were assumed to have achieved statistical cure. Population morbidity and mortality estimates were scaled up for the screen eligible cohort. Morbidity was increased by $25 \%$ and mortality was scaled up to account for smoking history, and additionally for smoking intensity. The full methods for this adjustment are available elsewhere. ${ }^{17}$

\section{Model inputs}

Model parameters were determined by a combination of literature reviews, parameters used in previous models published in peer-reviewed literature, and analyses of NZ-linked health datasets. Intervention parameters were primarily and preferentially sourced from the NELSON trial. ${ }^{1526} 27$ For each parameter, we determined a point estimate and distribution for use in probabilistic uncertainty analysis (table 1 ).

Several key parameters in the original $\mathrm{BODE}^{3}$ model were updated (table 1) by incorporating findings from the recent NELSON RCT, where a more detailed nodule management protocol (using nodule volumes and volume doubling time) improved the specificity of screening in identifying lung cancer, reduced the number of diagnostic tests required and resulted in a small $(2 \%)$ increase in the number of CT scans. ${ }^{15}$ Other parameter updates include stage at diagnosis, stage-specific survival, CT scan costs, diagnostic test adherence and overdiagnosis (described in online supplemental methods).

We set screening coverage in the base case to be equal for Māori and non-Māori, thereby setting an expectation for equal treatment by ethnicity. A well-designed programme that prioritises Māori engagement and outcomes can achieve high screening coverage for Māori without any additional costs. ${ }^{28} \mathrm{~A}$ scenario analysis of increased costs to address inequities in screening coverage due to a poorly designed programme was also undertaken by doubling invitation costs for the entire screening cohort.

Lead time bias was accounted for by delaying excess mortality for screen-detected cancers by 6 months, with sensitivity analyses of varying lengths of lead time. Overdiagnosis was included by removing $8.9 \%$ of screen-detected local stage cancers in the LDCT arm. ${ }^{8}$

Our primary analyses included background (ie, nonlung cancer) mortality rates and expected background morbidity in the main life table. Morbidity was estimated by sex, ethnic group and age, using the total years of life lived with disabilities (YLDs) from an NZ Burden of Disease Study, ${ }^{29}{ }^{30}$ divided by the population count in the same group. Health gain could only occur in the envelope of 'good health' that reduced with increasing age and was less for Māori. For example, a Māori woman aged 60-64 years has an expected YLD of 0.88 , meaning a year of life gained in this population group has a maximum utility value of 0.712 .

Within the model we used invitation costs from the NZ colorectal cancer screening pilot study ${ }^{31}$ and applied them evenly across population groups. This spread the cost of any activities to increase screening coverage in specific groups (such as Māori) equally across the entire population-so as not to penalise under screened populations for the limitations of the current health system. Excess costs (ie, excess to that expected for an 'average' NZ citizen of the same sex and age ${ }^{32}$ and cost offsets (health system costs averted as a result of screening) varied for being in the first year of lung cancer diagnosis, remission or last 6 months of life if dying of lung cancer.

\section{Analyses}

Our base case analysis assumes equal intervention coverage, equal sensitivity and specificity of LDCT in detecting lung cancer, and equal per capita intervention and health system costs for Māori and non-Māori, with ethnic specific-estimates of the eligible population, lung cancer incidence, stage at diagnosis, lung cancer net survival, and background morbidity and mortality. The model was run as a Monte Carlo simulation with 2000 iterations, each drawing randomly from the range around each input parameter to produce a modelled best estimate with a $95 \%$ uncertainly interval (UI). The primary model outputs include health gains (HALYs total and per capita), baseline and intervention costs, incremental cost-effectiveness ratio (ICER) and equity analyses as described below. To allow the direct comparison of Māori and non-Māori health gains, the per capita health gains are age standardised to the 2001 Māori age standard. ${ }^{33}$ Tornado plots were constructed to examine the impact of uncertainty in each input parameter on uncertainty in the output ICER, by rerunning the model with the 2.5th and 97.5th percentile values of the input parameters (online supplemental figures 1-3).

We measured relative and absolute inequalities in lung cancer mortality and HALE before and after the intervention for the 55-59-year-old model cohort (as this group would receive the full screening experience over their lifetime). We also calculated the absolute difference in lives saved and healthy days gained for Māori compared with non-Māori by subtracting the baseline difference in mortality and HALE from the intervention mortality and HALE difference. The absolute change in inequality in HALE was then converted to healthy days (by multiplying by 365). These were calculated for the 55-59year screeneligible cohort and were also weighted up to measure these gains over the total population in this age group by 
Table 1 Input parameters for modelling LDCT lung cancer screening in the New Zealand (NZ) setting

\begin{tabular}{lllll}
\hline Variable & $\begin{array}{l}\text { Base case } \\
\text { (range) }\end{array}$ & $\begin{array}{l}\text { Distribution } \\
\text { Performance of CT as a screening test }\end{array}$ & $\begin{array}{l}\text { Source in this } \\
\text { model }\end{array}$ & $\begin{array}{l}\text { Change with } \\
\text { respect to BODE }\end{array}$ \\
model
\end{tabular}

\section{Performance of diagnostic tests}

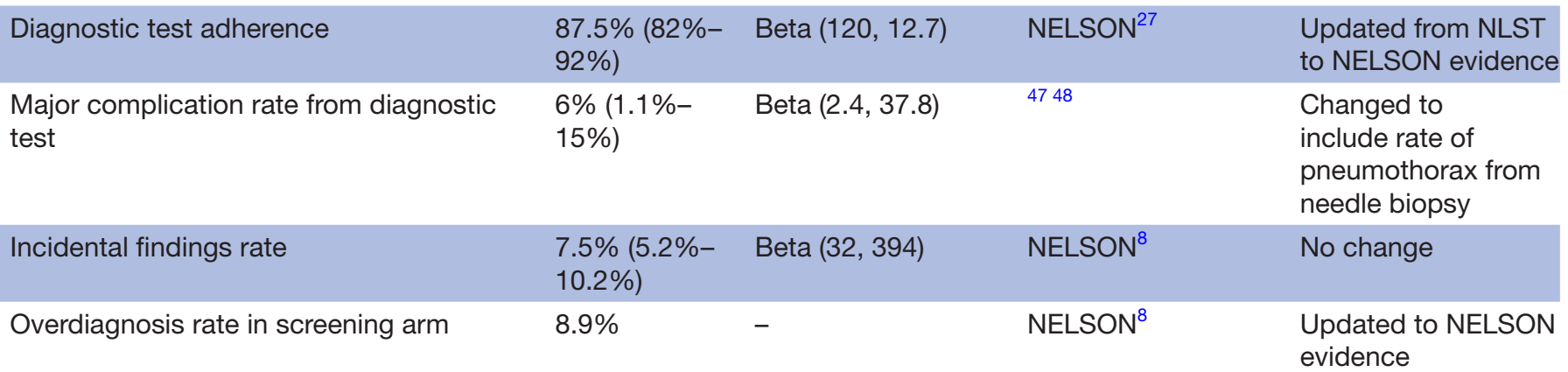

\section{Disease/state morbidity}

DW for first 5 months lung cancer diagnosis 0.469 and treatment

\section{Nil}

Nil

Nil

Based on GBD DWs No change with disaggregation by clinical phase 2949

\begin{tabular}{|c|c|c|c|c|}
\hline DW for 1 month assumed terminal & 0.548 & Nil & 2949 & No change \\
\hline $\begin{array}{l}\text { DW for } 5 \text { months assumed preterminal lung } \\
\text { cancer }\end{array}$ & 0.539 & Nil & 2949 & No change \\
\hline $\begin{array}{l}\text { DW per annum after diagnosis and } \\
\text { treatment (ie, remission) }\end{array}$ & 0.315 & Nil & 2949 & No change \\
\hline DW for complication from diagnostic test & 0.0158 & Nil & 2949 & $\begin{array}{l}\text { No change (based } \\
\text { on moderate } \\
\text { respiratory disease } \\
\text { for } 30 \text {-day duration) }\end{array}$ \\
\hline
\end{tabular}

Incidence, stage at diagnosis, mortality and survival rates

\begin{tabular}{|c|c|c|c|c|}
\hline $\begin{array}{l}\text { Lung cancer stage at } \\
\text { diagnosis (\%) }\end{array}$ & $\begin{array}{l}\text { Local } \\
\text { Regional } \\
\text { Distant }\end{array}$ & $\begin{array}{l}13 \\
23.3 \\
63.7\end{array}$ & Nil & $\begin{array}{l}\text { MLCR (online } \\
\text { supplemental } \\
\text { appendix A, online } \\
\text { supplemental file) }\end{array}$ \\
\hline
\end{tabular}

\begin{tabular}{|c|c|c|c|c|c|}
\hline $\begin{array}{l}\text { Stage distribution } \\
\text { with LDCT lung } \\
\text { screening (\%) }\end{array}$ & $\begin{array}{l}\text { Local } \\
\text { Regional } \\
\text { Distant }\end{array}$ & $\begin{array}{l}66.3 \\
21.6 \\
12.1\end{array}$ & Nil & NELSON $^{27}$ & $\begin{array}{l}\text { Updated from the } \\
\text { proportionate shift } \\
\text { observed in NLST } \\
\text { to a final distribution } \\
\text { that matches } \\
\text { NELSON }\end{array}$ \\
\hline
\end{tabular}

Continued 
Table 1 Continued

\begin{tabular}{|c|c|c|c|}
\hline Variable & $\begin{array}{l}\text { Base case } \\
\text { (range) }\end{array}$ & Distribution & $\begin{array}{l}\text { Source in this } \\
\text { model }\end{array}$ \\
\hline Lung cancer relative survival & $\begin{array}{l}\text { Varied by sex, } \\
\text { age, ethnicity } \\
\text { and stage }\end{array}$ & Nil & $\begin{array}{l}\text { From analyses } \\
\text { of linked cancer } \\
\text { mortality data } \\
\text { by stage and } \\
\text { operationalised as } \\
\text { log-normal survival } \\
\text { probabilities (online } \\
\text { supplemental } \\
\text { appendix A, online } \\
\text { supplemental file). }\end{array}$ \\
\hline
\end{tabular}
Change with
respect to $\mathrm{BODE}^{3}$
model

\section{Changed to} stage-specific Pohar Perme net survival estimates derived from analysis of NZCR data where SEER extent of disease was available or calculated from TNM values

\begin{tabular}{|c|c|c|c|c|}
\hline $\begin{array}{l}\text { Background (ie, non-lung) mortality with } \\
1.75 \% / 2.25 \% \text { annual decrease for non- } \\
\text { Māori/Māori }\end{array}$ & $\begin{array}{l}\text { Varied by sex, } \\
\text { ethnicity and } \\
\text { age }\end{array}$ & Nil & $\begin{array}{l}\text { From projected life } \\
\text { tables by sex, age } \\
\text { and ethnicity }{ }^{50}\end{array}$ & No change \\
\hline Background or expected morbidity & $\begin{array}{l}\text { Varied by sex, } \\
\text { ethnicity and } \\
\text { age }\end{array}$ & Nil & $\begin{array}{l}\text { Prevalent YLDs from } \\
\text { NZ BDS }\end{array}$ & No change \\
\hline Cessation rate & $\begin{array}{l}\text { Varied by sex, } \\
\text { ethnicity and } \\
\text { age }\end{array}$ & Nil & $\begin{array}{l}\text { 'Pessimistic } \\
\text { scenario' projections } \\
\text { from analyses of } \\
2006-2013 \text { census } \\
\text { data }^{25}\end{array}$ & $\begin{array}{l}\text { 'Best estimate' } \\
\text { projections from } \\
\text { census data }\end{array}$ \\
\hline Lead time (years) & 0.5 & Nil & 51 & No change \\
\hline \multicolumn{5}{|l|}{ Direct costs (all in NZ\$) } \\
\hline Cost per person invited & $30(19-43)$ & Gamma $(25,1.2)$ & 52 & \\
\hline $\begin{array}{l}\text { Percentage increase in CT costs to account } \\
\text { for new nodule monitoring protocol (\%) }\end{array}$ & $2(1.1-3.1)$ & Beta $(15,737)$ & NELSON RCT ${ }^{15}$ & $\begin{array}{l}2 \% \text { additional scans } \\
\text { for nodules }\end{array}$ \\
\hline Cost per diagnostic test & $\begin{array}{l}1837(1214- \\
2622)\end{array}$ & Gamma $(25,73)$ & 52 & No change \\
\hline Cost of incidental findings & $500(324-714)$ & Gamma $(25,20)$ & $\begin{array}{l}{ }^{17} \text { (online } \\
\text { supplemental } \\
\text { appendix A, online } \\
\text { supplemental file). }\end{array}$ & No change \\
\hline Cost of major complications & $\begin{array}{l}2835(1820- \\
4148)\end{array}$ & Gamma $(25,113)$ & $\begin{array}{l}\text { Based on purchasing } \\
\text { power parity } \\
\text { adjusted }^{53} \text { cost of a } \\
\text { pneumothorax }^{54}\end{array}$ & No change \\
\hline
\end{tabular}

*Excess to 'average' NZ.

$\mathrm{BODE}^{3}$, Burden of Disease Epidemiology, Equity and Cost-Effectiveness Programme; DW, disability weight; GBD, Global Burden of Disease; LDCT, low-dose CT; MLCR, Midland Lung Cancer Registry; NZCR, New Zealand Cancer Registry; RCT, randomised controlled trial; SEER, Surveillance, Epidemiology and End Results programme of the National Cancer Institute; TNM, tumour, node, metastasis cancer staging; YLD, year lost due to disability. 
multiplying by the proportion eligible for screening out of the total population and dividing by the total population in the age group in 2011.

A number of sensitivity analyses were also run including equity-focused analyses where: (1) we replaced Māori data with non-Māori values for baseline mortality and morbidity to address the issues that (A) lower life expectancy of Indigenous populations results in less opportunity for life-years gained with screening and (B) that lower life expectancy is, in part, a result of lung cancer inequities and (2) we assessed the impact of improvements in treatment for Māori following the implementation of a screening programme, given evidence of greater delays to treatment for Māori in the current health system compared with non-Māori leading to poorer survival. ${ }^{34-36}$ For this last scenario, for Māori who are screened, we replaced Māori age-specific excess mortality from lung cancer with the non-Māori rate. This scenario ignores any improvements to non-Māori survival and likely overestimates the improvements for Māori by ignoring the influence of comorbidities on treatment and survival.

Other sensitivity analyses examined variations in key input parameters of the costs of the CT scan, complication rate, overdiagnosis, lead time, invitation costs (to account for increased costs of increasing Māori screening coverage), screening cost weight to account for additional CT scans associated with the nodule management protocol ${ }^{37}$ and discount rate.

\section{RESULTS}

The modelled CT lung cancer screening intervention for smokers aged 55-74years resulted in health benefits for all population groups and was cost-effective for all modelled groups (using an ICER threshold of NZ $\$ 45000$ per HALY). The ICERs ranged from NZ $\$ 24700$ per HALY (95\% UI NZ\$19900 to NZ\$30400) for Māori females up to NZ\$39100 per HALY for non-Māori males (95\% UI NZ\$31400 to NZ\$48900). The size of the health gains from the intervention was greater for Māori compared with non-Māori and for women compared with men (table 2). Māori health gains (HALYs per capita) were approximately two times greater for Māori females compared with non-Māori females (0.112 HALYs/capita, $95 \%$ UI 0.080 to 0.151 and $0.063,95 \%$ UI 0.045 to 0.083 , respectively), and $25 \%$ greater for Māori males compared with non-Māori males $(0.068,95 \%$ UI 0.048 to $0.092 \mathrm{cf}$ $0.051,95 \%$ UI 0.037 to 0.069 ) with overlap in the UIs (table 2).

The modelled LDCT screening intervention is highly likely to be cost-effective for all sex and ethnic groups, given a willingness to pay threshold based on GDP per

Table 2 Lifetime costs, HALYs and ICERs (95\% uncertainty intervals) for biennial CT screening of smokers aged 55-74years with a 30 pack-year smoking history, by ethnic group and gender, among the 2011 population

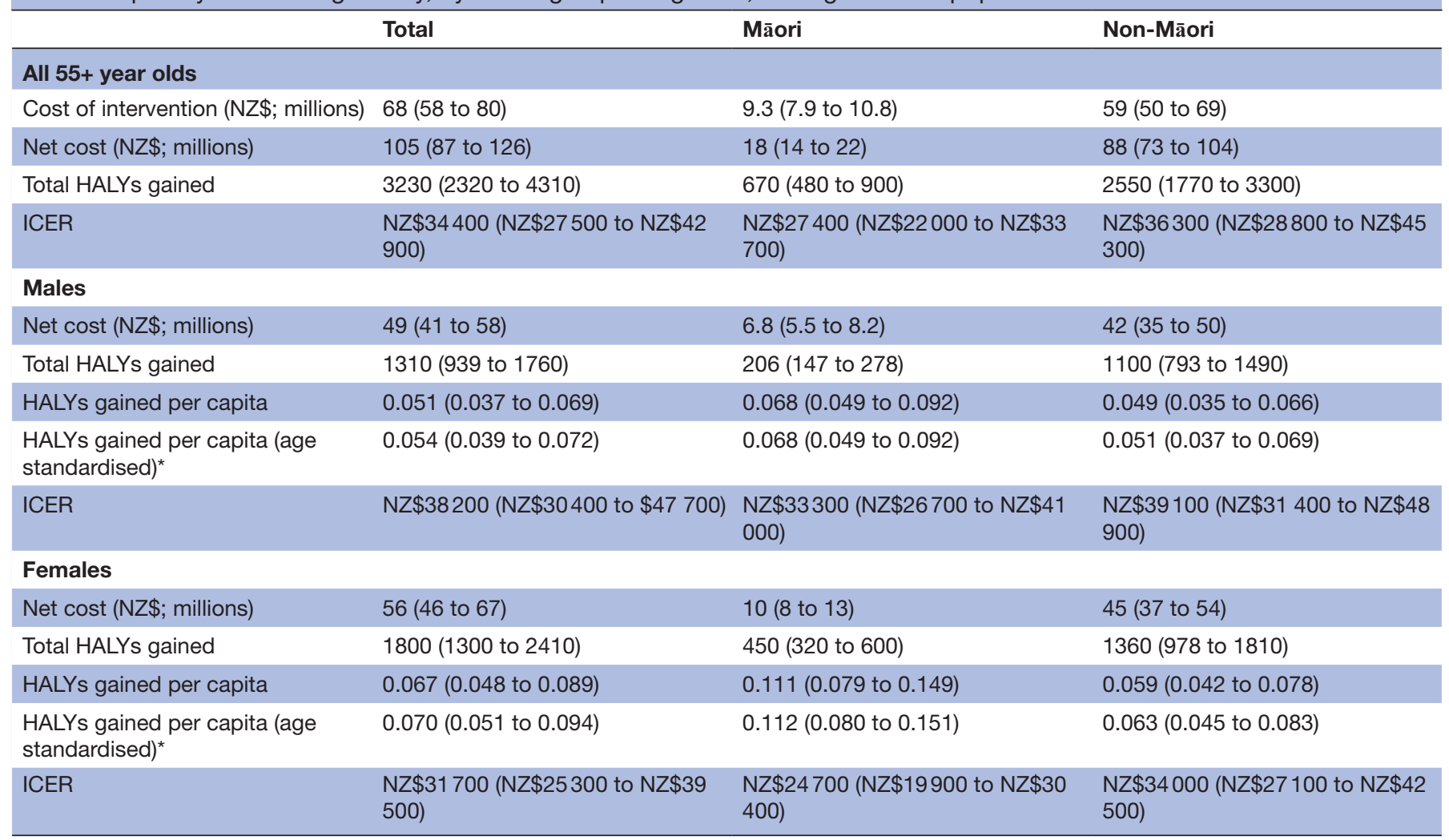

Discount rate $3 \%$.

*Age standardised to the 2001 Māori population standard.

HALY, health-adjusted life-year; ICER, incremental cost-effectiveness ratio. 


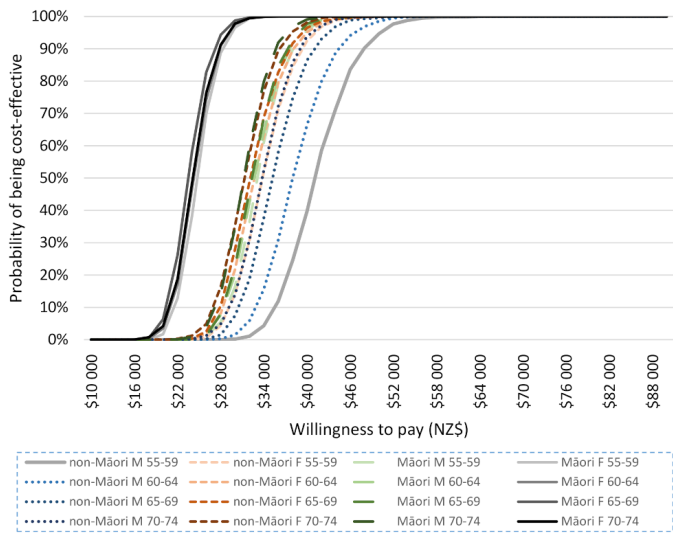

Figure 2 Cost-effectiveness acceptability curve for lowdose CT screening for lung cancer, by age group, gender (male (M), female (F)) and ethnicity.

capita (eg, NZ\$45000 per HALY) (figure 2). LDCT screening is likely to be relatively more cost-effective for Māori than for non-Māori, for females than for males, and for those in the older eligible age group (70-74 years) compared with the younger eligible age groups (55-59 years) (figure 2) LDCT screening remained cost-effective for the total population in all but one of the sensitivity analyses on key parameters (table 3 ).

Substituting non-Māori background mortality for Māori background mortality provided a $25 \%$ increase in Māori HALY gains compared with the default model (820 cf 660 HALYs; table 3). Using values of non-Māori background morbidity had a smaller gain in HALYs for Māori, with a $14 \%$ increase in total HALYs relative to the default model. The combination of setting non-Māori levels of background morbidity and mortality for Māori in the model resulted in a $42 \%$ additional total HALYs for Māori over default. Improving stage-specific survival for Māori to the level of non-Māori resulted in a $36 \%$ increase in Māori total HALYs. In all these scenarios, there was little change to the costs and therefore changes to the ICERs were primarily driven by changes in the health gains.

\section{Impacts on inequalities in lung cancer survival and absolute} inequalities in HALE

For the 55-59-year-old cohort, Māori had higher baseline lung cancer mortality than non-Māori. Mortality rates per 1000 eligible population reduced for both Māori and non-Māori with LDCT lung screening, and there was a reduction in the rate difference between baseline and intervention, indicating a reduction in absolute mortality inequities with screening (table 4). For Māori males, the reduction in absolute inequity in lung cancer mortality resulted in an additional 1.4 (95\% UI 1.2 to 1.7 ) lives saved per 1000 eligible population for Māori males over the reduction in mortality for non-Māori males. Weighted to spread this gain over the total population aged 55-59 years, this reduced to an additional 0.5 (95\% UI 0.4 to 0.6) lives saved per 1000 population for Māori males. The gain in lives saved were even greater for Māori females, with an additional 5.6 (95\% UI 4.4 to 6.9 ) lives saved per
1000 eligible population, and 1.1 (95\% UI 0.8 to 1.4 ) lives saved per 1000 total population over the mortality gains for non-Māori females. In contrast, the relative inequity in mortality for the eligible population increased slightly, with overlapping UIs (table 4). Using a scenario of equal stage-specific survival, there were bigger gains in health equity as measured by changes to lung cancer mortality and in lives saved for Māori over non-Māori (online supplemental table S1).

For HALE, Māori baseline and intervention HALE were lower than non-Māori HALE, and both Māori and nonMāori HALE improved with screening (table 4). Absolute and relative inequities in HALE between Māori and non-Māori reduced slightly with CT lung screening. With LDCT screening, Māori females gained 20 healthy days over the gains of non-Māori females, and for Māori males there was a gain of 8 healthy days over non-Māori males. The scenario using equal stage-specific survival demonstrated greater gains in health equity for Māori over non-Māori (online supplemental table S1). For HALE in the eligible population, Māori females under this scenario gained 60 healthy days over non-Māori females and/or Māori males healthy days gained over non-Māori increased to 30 healthy days under this scenario (online supplemental table $\mathrm{S} 1$ ).

\section{DISCUSSION}

LDCT screening for lung cancer is likely to be cost-effective in the NZ setting and will likely contribute positively to reducing population level inequities in lung cancer for Māori. In the model, Māori achieve greater per capita health gains compared with non-Māori due to higher rates of tobacco use, and higher incidence and mortality of Māori from lung cancer. These greater health gains for Māori translate into more favourable cost-effectiveness for this population compared with the total population.

Previous international estimates of the cost-effectiveness of LDCT lung cancer screening have been mixed. ${ }^{10131738-40}$ Similar to other studies, we found varying health gains and cost-effectiveness by demographic grouping, with females, older eligible participants and Māori likely to achieve greater gains in health and be relatively more cost-effective than males, the youngest eligible modelled cohort, and non-Māori, respectively. ${ }^{17} 41$ The BODE 2018 cost-effectiveness analysis ${ }^{17}$ concluded that lung cancer screening was unlikely to be cost-effective in NZ, and this conclusion impacted policy discussions regarding lung cancer screening in NZ. ${ }^{17}$ A recent correction to this paper now finds CT lung cancer screening is costeffective for Māori. ${ }^{18}$ This differs from our finding that LDCT screening is likely to be cost-effective for all eligible groups in NZ. The major drivers of the improved estimates of cost-effectiveness in our model compared with the $\mathrm{BODE}^{3}$ model are our updates to stage shift, improved sensitivity and specificity reported in the NELSON RCT, and lower CT costs. 
Table 3 Scenario analyses for the total eligible population and equity scenarios for Māori (95\% uncertainty intervals)

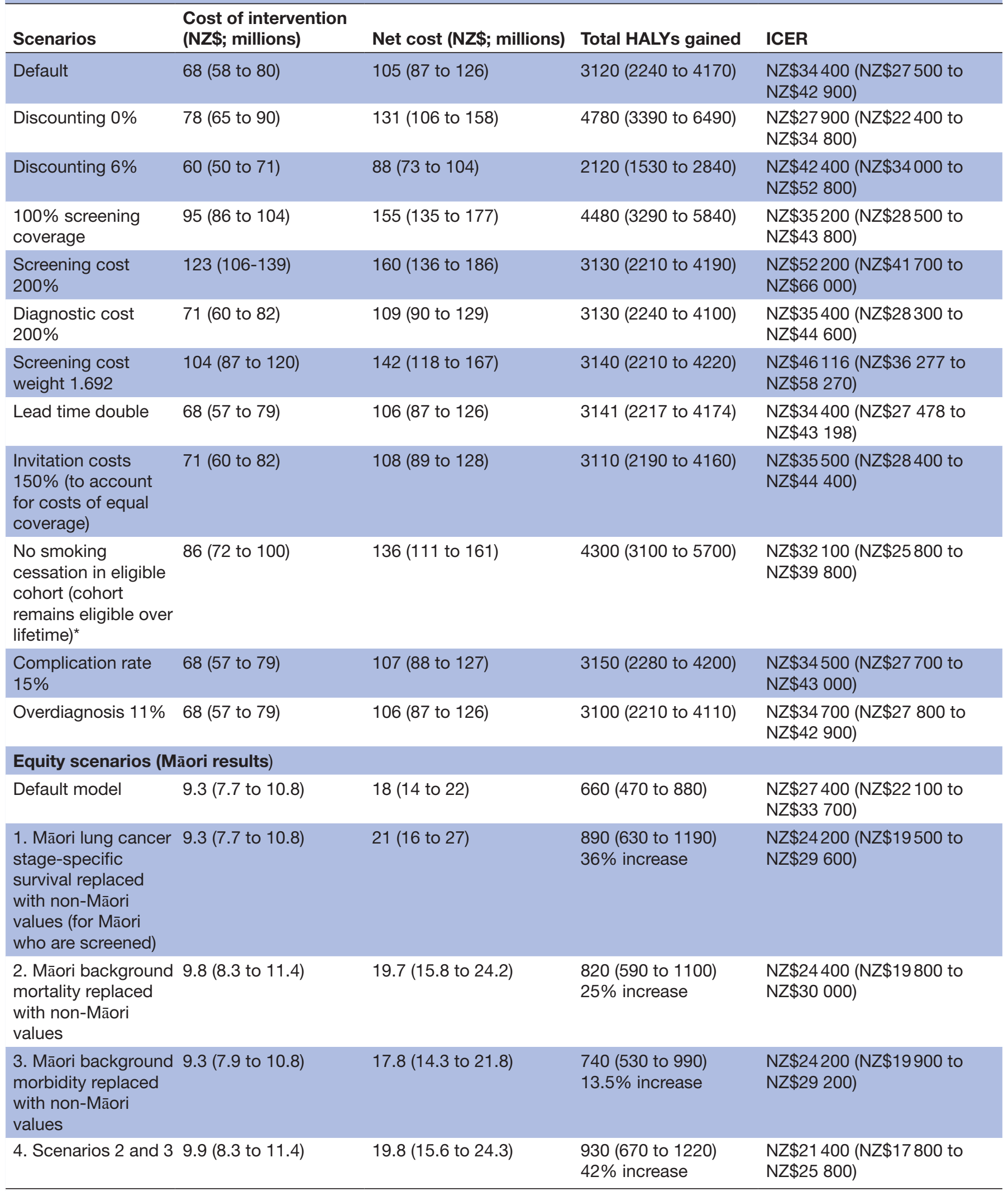

${ }^{*}$ Costs associated with background morbidity and mortality were adjusted to reflect the altered age structure of the eligible cohort. HALY, health-adjusted life-year; ICER, incremental cost-effectiveness ratio. 


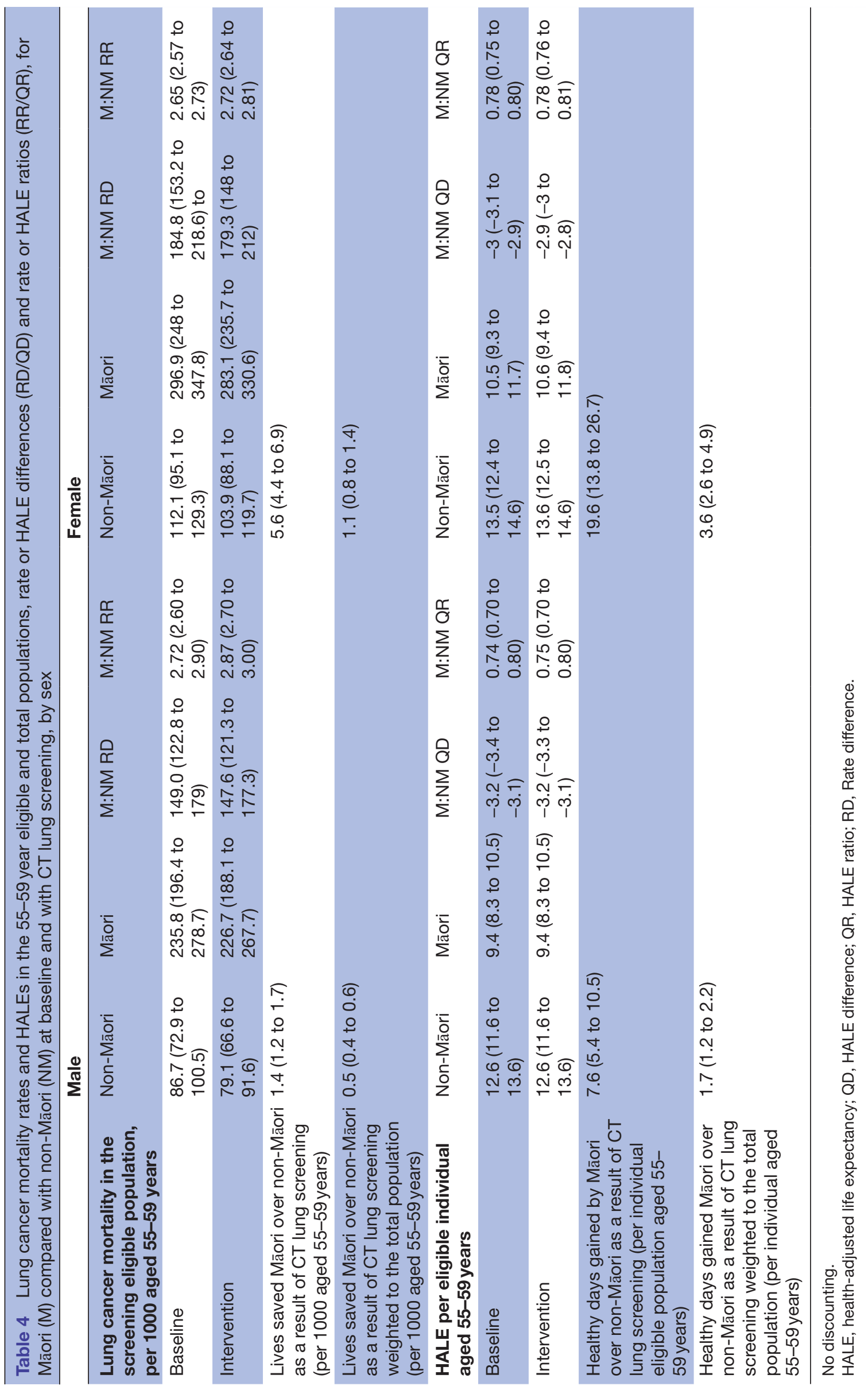


Our equity-focused scenarios demonstrated the important impact of existing inequities in health and healthcare for Māori on modelled health gains from LDCT screening for lung cancer. Removing the penalty of having lower life expectancy and a higher burden of comorbid conditions for Māori resulted in a large increase in per capita HALY gains (increased by $42 \%$ ) and also a reduction in the ICER for Māori, further improving the cost-effectiveness profile. An improvement in HALYs per capita (36\% increase over default level) and cost-effectiveness for Māori was also seen from the scenario of equal stage-specific survival, where Māori who engaged with screening achieved the same levels of stagespecific survival as non-Māori. This scenario assumes that inequities in access to and quality of care for lung cancer for Māori will disappear with a structured and monitored screening programme, thereby equalising stage-specific survival. This scenario ignores the contribution of other factors to stage-specific survival inequities, such as comorbidities, any ethnic differences in the proportion of the distribution of small cell lung cancers that have worse prognosis and assumes that improvements in access and care for Māori will mitigate both the existing inequities and any improvements to non-Māori that may also arise from increased standardisation of care.

A strength of our approach to modelling was the primacy of health equity for Māori in all of our modelling decisions. In addition to building several equity-specific model outputs (such as changes to mortality and HALE inequities), we gave priority to updating model parameters that were likely to have an important impact on health equity (regardless of whether this impact was positive or negative). In this paper, we included new estimates of Surveillance, Epidemiology and End Results programme of the National Cancer Institute extent of disease at diagnosis drawn from a registry which has provided almost complete extent of disease at diagnosis. Importantly, this analysis showed that there are no measurable differences in extent of disease at diagnosis for Māori compared with non-Māori which is consistent with the published analysis of stage at diagnosis from that source. ${ }^{16}$

We also included new estimates of stage-specific survival that show a large inequity for localised lung cancer in Māori compared with non-Māori. This is an important finding in the context of LDCT lung cancer screening which primarily aims to increase the proportion of localised cancers. Because stage at diagnosis does not differ for Māori and non-Māori, worse stage-specific survival for localised lung cancer results in an increase in relative mortality inequities for Māori (compared with nonMāori) in the screen eligible population. Should LDCT lung cancer screening be implemented in NZ, it will be critical to address this differential stage-specific survival for Māori by addressing access to and quality of healthcare for those diagnosed with lung cancer alongside the appropriate management of comorbid conditions.

In our model, we set the expectation that a new screening programme would achieve equal coverage for
Māori and non-Māori, thereby not extending existing inequities in screening service access. There are examples of health service providers in NZ that have achieved equal or greater screening coverage for Māori, demonstrating that, with good design and engagement with Māori, equal coverage is achievable. ${ }^{28} 42$ Our scenario analysis of increased invitation costs to improve Māori screening coverage remained cost-effective. In the model we account for outflow, where a proportion of the population will reach 15 years post cessation after initially being eligible. However, we do not account for any inflow into the model, where those initially ineligible subsequently acquire a 30 pack-year smoking history. As a result, our estimates of health gains and cost-effectiveness may be conservative. We used the NLST eligibility criteria for a higher risk eligible population, for consistency with previous NZ-based analyses. Using our model, the wider eligibility criteria of NELSON gives greater total HALYs gains, but similar ICERs due to the balancing impacts of the lower risk of lung cancer (lower per capita HALY gains) in a younger cohort (greater per capita HALY gains).

From an equity perspective, an important limitation of our LDCT screening model was the sole focus on lung cancer, to produce a conservative estimate of the possible health gains, the impacts on inequities for Māori and the cost-effectiveness. There is evidence that LDCT scans undertaken in such a high-risk population (30 pack-year smoking history) may offer potential co-benefits for the diagnosis and management of other health conditions, such as coronary artery calcification ${ }^{43}$ and chronic obstructive pulmonary disease, which are important conditions for the total population and have a greater burden on Māori. In addition, we did not include any changes to smoking behaviours that might occur as a result of LDCT screening, despite evidence that there can be an effect on cessation rates for those who engage in screening and that this impacts positively on cost-effectiveness models. ${ }^{40} 44$

We expect that this work will lead to further discussions regarding lung cancer screening in NZ. Furthermore, the methods we have used to ensure this cost-effectiveness analysis explicitly considered equity can be used in modelling for other conditions.

\section{CONCLUSIONS}

A national LDCT screening programme for lung cancer in NZ is likely to be cost-effective and will improve total population health. A well-planned and monitored programme can make an important contribution to reducing inequities in the burden of lung cancer for the Māori population. For these reasons, we highly recommend consideration of an LDCT screening programme in NZ.

Twitter Sue Crengle @tiekemanu

Acknowledgements We thank University of Otago, BODE ${ }^{3}$ for providing a copy of their original CT screening model. We also acknowledge the collaboration of Ross 
Lawrenson and his team at Waikato University who provided access to the Midland Lung Cancer Register for the purpose of determining baseline stage distribution in unscreened lung cancer among Māori and non-Māori.

Contributors All authors contributed to the conception or design of the work and the interpretation of data, give final approval for publication and agree to be accountable for all aspects of the work. MM, GK and PS undertook data analysis and modelling. MM drafted the manuscript. GK, PS, KB and SC revised the manuscript.

Funding This work was supported by the Waitematā and Auckland District Health Boards.

Competing interests GK and MM report personal fees from Waitematā District Health Board during the conduct of the study. GK was also a coauthor on the prior CT lung publication (Jaine et al, 2018) and received grants from the NZ Health Research Council outside the submitted work.

Patient consent for publication Not required.

Provenance and peer review Not commissioned; externally peer reviewed.

Data availability statement Some data may be obtained from a third party and are not publicly available. Some data for this study were sourced from the Ministry of Health, New Zealand, and are available from them by request.

Open access This is an open access article distributed in accordance with the Creative Commons Attribution Non Commercial (CC BY-NC 4.0) license, which permits others to distribute, remix, adapt, build upon this work non-commercially, and license their derivative works on different terms, provided the original work is properly cited, appropriate credit is given, any changes made indicated, and the use is non-commercial. See: http://creativecommons.org/licenses/by-nc/4.0/.

\section{ORCID iDs}

Melissa McLeod http://orcid.org/0000-0002-5834-7846

Sue Crengle http://orcid.org/0000-0001-9367-1492

\section{REFERENCES}

1 Ministry of Health. Cancer: new registrations and deaths 2013. Wellington: Ministry of Health, 2016.

2 Ministry of Health. New cancer registrations 2016. Wellington: Ministry of Health, 2018.

3 Allemani C, Matsuda T, Di Carlo V, et al. Global surveillance of trends in cancer survival 2000-14 (CONCORD-3): analysis of individual records for 37513025 patients diagnosed with one of 18 cancers from 322 population-based registries in 71 countries. Lancet 2018;391:1023-75.

4 Sandiford P, Abdel-Rahman ME, Allemani C, et al. How many cancer deaths could New Zealand avoid if five-year relative survival ratios were the same as in Australia? Aust N Z J Public Health 2015;39:157-61.

5 Aberle DR, Adams AM, et al, National Lung Screening Trial Research Team. Reduced lung-cancer mortality with low-dose computed tomographic screening. N Engl J Med 2011;365:395-409.

6 Oudkerk M, Devaraj A, Vliegenthart R, et al. European position statement on lung cancer screening. Lancet Oncol 2017;18:e754-66.

7 de Koning H, Van Der Aalst C, ten Haaf K, et al. PL02.05 effects of volume CT lung cancer screening: mortality results of the Nelson Randomised-Controlled population based trial. Journal of Thoracic Oncology 2018;13:S185

8 de Koning HJ, van der Aalst CM, de Jong PA, et al. Reduced lungcancer mortality with volume CT screening in a randomized trial. $N$ Engl J Med 2020;382:503-13.

9 National Lung Screening Trial Research Team. Lung cancer incidence and mortality with extended follow-up in the National lung screening trial. J Thorac Oncol 2019;14:1732-42.

10 ten Haaf K, Tammemägi MC, Bondy SJ, et al. Performance and cost-effectiveness of computed tomography lung cancer screening scenarios in a population-based setting: a Microsimulation modeling analysis in Ontario, Canada. PLoS Med 2017;14:e1002225.

11 Goffin JR, Flanagan WM, Miller AB, et al. Cost-Effectiveness of lung cancer screening in Canada. JAMA Oncol 2015;1:807-13.

12 Criss SD, Cao P, Bastani M, et al. Cost-Effectiveness analysis of lung cancer screening in the United States: a comparative modeling study. Ann Intern Med 2019;171:796-804.

13 Raymakers AJN, Mayo J, Lam S, et al. Cost-Effectiveness analyses of lung cancer screening strategies using low-dose computed tomography: a systematic review. Appl Health Econ Health Policy 2016;14:409-18.
14 Cressman S, Peacock SJ, Tammemägi MC, et al. The costeffectiveness of high-risk lung cancer screening and drivers of program efficiency. J Thorac Oncol 2017;12:1210-22.

15 Horeweg N, Scholten ET, de Jong PA, et al. Detection of lung cancer through low-dose CT screening (NELSON): a prespecified analysis of screening test performance and interval cancers. Lancet Oncol 2014;15:1342-50.

16 Lawrenson R, Lao C, Brown L, et al. Characteristics of lung cancers and accuracy and completeness of registration in the New Zealand cancer registry. $N$ Z Med J 2018;131:13-23.

17 Jaine R, Kvizhinadze G, Nair N, et al. Cost-Effectiveness of a lowdose computed tomography screening programme for lung cancer in New Zealand. Lung Cancer 2018;124:233-40.

18 Jaine R, Kvizhinadze G, Nair N, et al. Cost-Effectiveness of a lowdose computed tomography screening programme for lung cancer in New Zealand. Lung Cancer 2020;144:99-106.

19 Te Tiriti O Waitangi (the Treaty of Waitangi). 1840.

20 Ministry of Health. Background Information: New Zealand's Tobacco Control Programme. Wellington: Ministry of Health, 2016.

21 Blakely T, Fawcett J, Hunt D, et al. What is the contribution of smoking and socioeconomic position to ethnic inequalities in mortality in New Zealand? Lancet 2006;368:44-52.

22 Teng AM, Atkinson J, Disney G, et al. Ethnic inequalities in cancer incidence and mortality: census-linked cohort studies with 87 million years of person-time follow-up. BMC Cancer 2016;16:755.

23 Blakely T, Wilson N, BODE3 Team. Burden of Disease Epidemiology, Equity and Cost-Effectiveness $\left(B O D E^{3}\right)$ Study Protocol. Version 2.1. Wellington: Department of Public Health, University of Otago, 2012.

24 Hutubessy R, Chisholm D, Edejer TT-T. Generalized costeffectiveness analysis for national-level priority-setting in the health sector. Cost Eff Resour Alloc 2003;1:8.

25 van der Deen FS, Ikeda T, Cobiac L, et al. Projecting future smoking prevalence to 2025 and beyond in New Zealand using smoking prevalence data from the 2013 census. N Z Med J 2014;127:71-9.

$26 \mathrm{Ru} Z$ Zhao Y, Xie X, de Koning HJ, et al. Nelson lung cancer screening study. Cancer Imaging 2011;11 Spec No A:S79-84.

27 Yousaf-Khan $U$, van der Aalst $C$, de Jong PA, et al. Final screening round of the Nelson lung cancer screening trial: the effect of a 2.5year screening interval. Thorax 2017;72:48-56.

28 Sandiford P, Grey C, Salvetto M, et al. The population prevalence of undetected abdominal aortic aneurysm in New Zealand Māori. J Vasc Surg 2020;71:1215-21.

29 Salomon JA, Vos T, Hogan DR, et al. Common values in assessing health outcomes from disease and injury: disability weights measurement study for the global burden of disease study 2010 . Lancet 2012;380:2129-43.

30 Ministry of Health. Health loss in New Zealand 1990-2013: a report from the New Zealand burden of diseases, injuries and risk factors study. Wellington: Ministry of Health, 2016.

31 Litmus Limited. Evaluation of the bowel screening pilot: eligible population perspectives. Wellington: Ministry of Health, 2013.

32 Blakely T, Atkinson J, Kvizhinadze G, et al. Patterns of cancer care costs in a country with detailed individual data. Med Care 2015;53:302-9.

33 Robson B, Purdie G, Cram F, et al. Age standardisation - an indigenous standard? Emerg Themes Epidemiol 2007;4:3.

34 McLeod M, Blakely T, Kvizhinadze G, et al. Why equal treatment is not always equitable: the impact of existing ethnic health inequalities in cost-effectiveness modeling. Popul Health Metr 2014;12:12-15.

35 McLeod M, Kvizhinadze G, Boyd M, et al. Colorectal cancer screening: how health gains and cost-effectiveness vary by ethnic group, the impact on health inequalities, and the optimal age range to screen. Cancer Epidemiol Biomarkers Prev 2017;26:1391-400.

36 Stevens W, Stevens G, Kolbe J, et al. Ethnic differences in the management of lung cancer in New Zealand. $J$ Thorac Oncol 2008;3:237-44.

37 Field JK, Duffy SW, Baldwin DR, et al. The UK lung cancer screening trial: a pilot randomised controlled trial of low-dose computed tomography screening for the early detection of lung cancer. Health Technol Assess 2016;20:1-146.

38 Wade S, Weber M, Caruana M, et al. Estimating the costeffectiveness of lung cancer screening with low-dose computed tomography for high-risk smokers in Australia. $J$ Thorac Oncol 2018;13:1094-105.

39 Manser R, Dalton A, Carter R, et al. Cost-Effectiveness analysis of screening for lung cancer with low dose spiral CT (computed tomography) in the Australian setting. Lung Cancer 2005;48:171-85.

40 Villanti AC, Jiang Y, Abrams DB, et al. A cost-utility analysis of lung cancer screening and the additional benefits of incorporating smoking cessation interventions. PLoS One 2013;8:e71379. 
41 Black WC. Computed tomography screening for lung cancer in the National lung screening trial: a cost-effectiveness analysis. $J$ Thorac Imaging 2015;30:79-87.

42 Thomson RM, Crengle S, Lawrenson R. Improving participation in breast screening in a rural general practice with a predominately Maori population. N Z Med J 2009;122:39-47.

43 Gaudio C, Tanzilli A, Mei M, et al. Concomitant screening of coronary artery disease and lung cancer with a new ultrafast-low-dose computed tomography protocol: a pilot randomised trial. Sci Rep 2019;9:13872.

44 Brain K, Carter B, Lifford KJ, et al. Impact of low-dose CT screening on smoking cessation among high-risk participants in the UK lung cancer screening trial. Thorax 2017;72:912-8.

45 Ministry of Health. BSA New Zealand district health board coverage report: period ending 31 December 2019. Wellington Ministry of Health. 2020.

46 Litmus, Massey University, and Sapere Research Group. Interim evaluation report of the bowel screening pilot: screening round one; 2015.

47 Nanavaty P, Alvarez MS, Alberts WM. Lung cancer screening: advantages, controversies, and applications. Cancer Control 2014;21:9-14.
48 Wiener RS, Schwartz LM, Woloshin S, et al. Population-Based risk for complications after transthoracic needle lung biopsy of a pulmonary nodule: an analysis of discharge records. Ann Intern Med 2011;155:137-44.

49 Ministry of Health. Health loss in New Zealand: a report from the New Zealand burden of diseases, injuries and risk factors study, 20062016. Wellington Ministry of Health; 2013.

50 Statistics New Zealand. Census 2013 - Major Ethnic Groups in New Zealand. Wellington: Statistics New Zealand, 2013.

51 Wu D, Erwin D, Rosner GL. Sojourn time and lead time projection in lung cancer screening. Lung Cancer 2011;72:322-6.

52 Artus J, Love T, Blick G, et al. Evaluation of the New Zealand Bowel Screening Pilot Interim costing analysis - costs of the first screening round (2012-13). Wellington: Sapere Research Group, 2014.

53 OECD. PPPs and exchange rates: OECD national accounts statistics (database), 2019. Available: https://doi.org/10.1787/data-00004en[Accessed 11 Dec 2019].

54 Foster RT, Blakely T, Wilson N, et al. Protocol for direct costing of health sector interventions for economic modelling (including event pathways). In: Burden of Disease Epidemiology, Equity and CostEffectiveness Programme (BODE3). Wellington: University of Otago, 2012. 